\title{
Buckling and Post-buckling Behaviour of Prismatic Aluminium Columns Submitted to a Series of Compressive Loads
}

\author{
A. Ziółkowski • S. Imiełowski
}

Received: 30 July 2010 /Accepted: 6 December 2010 /Published online: 23 December 2010

(C) The Author(s) 2010 This article is published with open access at Springerlink.com

\begin{abstract}
The buckling and post-buckling behaviour of prismatic aluminium columns from stocky to very slender shapes is investigated. The unconventional, in terms of buckling tests, displacement control of compressive load and a series of loadings provided an enhanced insight into the buckling process. A phenomenon of buckling load drop has been detected in columns of intermediate slenderness, reaching over $20 \%$ of the load early critical value. This newly observed occurrence resembles finite disturbance instability, which until recently was commonly believed to only appear in cases of thin walled cylindrical shells, but not columns. The observation is in contradiction to predicted results from the elasto-plastic buckling models of Engesser or Shanley, with constant or growing values of load during the post-buckling process. Further tests on columns of intermediate slenderness, with strain gauges glued at node and anti-node locations of the buckled profiles, revealed that even minute buckling results in fields of highly non-symmetric residual microplastic strain. The results of the present study indicate that running column buckling tests under displacement control is worthy of being adopted as common practice. The envelope of column post-buckling states can be conveniently determined. This information will in turn allow for the quick and reliable estimation of the safety of a column, which has undergone accidental or deliberate damage in the form of limited buckling when under operational load.
\end{abstract}

\footnotetext{
A. Ziółkowski $(\square) \cdot$ S. Imiełowski

Institute of Fundamental Technological Research,

Polish Academy of Sciences,

ul. Pawińskiego 5B,

02-106 Warsaw, Poland

e-mail: aziolk@ippt.gov.pl

S. Imiełowski

e-mail: simiel@ippt.gov.pl
}

Keywords Aluminium column instability · Finite disturbance buckling · Buckling load drop · Post-buckling . Micro plastic strains · Non-symmetric residual stresses . Column design criteria

\section{Introduction}

The failure of structural elements through buckling has aroused, and still generates, vivid interest amongst researchers and engineers. The study of the buckling behaviour of prismatic columns is a convenient step towards understanding and evaluating the reliability of more complex structures. It is currently well known that the basic physical action causing the underlying buckling of axially compressed columns is the depletion of its lateral stiffness to a value of zero. A number of intricacies exist, which complicate the prediction of buckling loads in actual engineering situations. The Euler linear elastic model of the prismatic, simply supported column still remains the fundamental tool for investigations of buckling stability. It predicts that the column will buckle at load $P_{E}$ (see e.g. Simitses and Hodge [10]):

$$
\begin{aligned}
P_{E} & =E A \cdot \pi^{2} / \lambda_{e f}^{2}, \sigma_{E}=E \pi^{2} / \lambda_{e f}^{2}, \lambda_{e f}=L_{e f} / r, L_{e f} \\
& =k \cdot L, I=A \cdot r^{2},
\end{aligned}
$$

where $P_{E}$ denotes the Euler buckling load, $\sigma_{E}$ is the Euler buckling stress, $E$ is the Young's modulus, $A$ is the column cross section area, $\lambda_{e f}$ is the column effective slenderness (in the sequel subscript "ef" is skipped to simplify notation), $L$ is the column length, $k$ is the index depending on column support conditions $-k=1$ for pinned-pinned ended column, $k=1 / 2$ for fixed-fixed ended column, $I$ denotes the column cross section moment of inertia, and $r$ denotes the radius of inertia. 
Systematic works, both theoretical and experimental, are continuously undertaken, aimed at expanding the scope of knowledge on the structural stability of columns to embrace various types of nonlinearities. The problem of finding a buckling column post-critical equilibrium shape when large displacements are admissible, an Elastica problem, was already solved by Lagrange in the nineteenth century in terms of elliptic integrals. However, the stability of these post-buckling configurations was only recently investigated by Kuznetsov and Levyakov [7]. Garikipati et al. [4] extended the formulation of classic elastic column stability problems to include large strains in order to enable the study of soft biological tissues. Domokos et al. [2] considered the buckling of elastic columns with lateral defections constrained by rigid, frictionless side-walls both theoretically and experimentally, i. e. problems with nonlinearities arising due to contact with boundary conditions. Magnusson et al. [8] abandoned the commonly adopted assumption in the investigation of the post-buckling behaviour of compressed columns, of constant loading force in post-buckling equilibrium states, and investigated instead the behaviour of "extensible" elastica. Their study was further enhanced by the work of Mazzilli [9] who analytically approached the problem of extensible elastica by loading not only axial forces but also transverse forces and bending moments at the column ends, and found post-buckled configurations in a number of cases.

Structural design practice requires the incorporation of other factors besides material and geometrical nonlinearities, such as load-column system imperfections (e.g. initial curvature, non-uniformity of cross-section, eccentricity of load), heat affected zones, residual stresses or the interactions of various modes of deformation. Barbero et al. [1] experimentally studied the interaction of local (flange) and global (Euler) buckling modes of a column, and revealed that upon interaction of the mechanisms a tertiary combined mode of buckling develops. They showed that the critical load value of the combined mode is lower than that predicted by the classic single mode Euler buckling model and that it is highly sensitive to imperfections.

Nowadays in practice, the design of column-like structural elements largely relies on two modelling "design tools". The approach recommended by the Aluminium Association takes advantage of the less refined theory of the Tetmayer-Jasinski failure criterion for axially compressed columns, see Figure 5.16 in Kissel and Ferry [6]:

$\sigma_{T J}(\lambda)= \begin{cases}\sigma_{0.2} & 0 \leq \lambda \leq \lambda_{1} \\ \sigma_{P}+\frac{\left(\sigma_{0.2}-\sigma_{P}\right)}{\left(\lambda_{P}-\lambda_{1}\right)} \cdot\left(\lambda_{P}-\lambda\right) & \lambda_{1} \leq \lambda \leq \lambda_{P}, \quad \lambda_{P}=\pi \sqrt{E / \sigma_{P}} \\ \sigma_{E}(\lambda)=\pi^{2} E / \lambda^{2} & \lambda_{P}<\lambda\end{cases}$

where $\sigma_{T J}(\lambda)$ denotes the Tetmajer-Jasinski critical stress, $\sigma_{0.2}$ is the conventional plastic yield stress and $\sigma_{P}$ is the proportionality limit stress. The three regimes of com- pressed member failure are distinguished depending on its slenderness, in the range $0 \leq \lambda \leq \lambda_{1}$, where the primary failure mechanism is due to bulk plastic flow with the slenderness $\lambda_{1}$ determined experimentally for a specific material, in the range $\lambda_{1} \leq \lambda \leq \lambda_{P}$ where inelastic buckling is the dominant failure mechanism and the slenderness $\lambda_{P}$ denotes the end of theoretical validity of linear elastic Euler buckling model, and in the range $\lambda_{P}<\lambda$ were Euler elastic buckling is the failure mechanism.

The British Standards and AISC (American Institute of Steel Construction) employ the Ayrton-Perry formula as the basic tool to evaluate structural buckling safety:

$\left(\sigma_{0.2} / \sigma_{A P}-1\right) \cdot\left(1-\sigma_{A P} / \sigma_{E}\right)=\eta$

where $\sigma_{A P}(\lambda)$ denotes the Ayrton-Perry critical stress, and $\eta$ is the dimensionless Perry "imperfection" factor characterising the column-load system "imperfection". On purely theoretical grounds, formula (3) is only valid for a column with initial shape imperfections in the form of a half sine function $f_{0}(x)=\delta_{0} \sin (\pi x / L) ; x \in<0, L>$. Detailed derivation and underlying assumptions of the formula counterpart to (3) can be found in Simitses and Hodge [10]. The Perry factor has the following exact interpretation $\eta=$ $\delta_{0} \cdot\left(h / r^{2}\right)$ in this case, where $\delta_{0}$ is the initial lateral deflection of the column centre, and $h$ denotes the extreme fibre distance from the centroidal axis in the column cross section. When imperfections of the load-column system take the form of an eccentricity of load, the Ayrton-Perry formula takes the following form:

$\left(\sigma_{0.2} / \sigma_{A P}-1\right) \cos \left(\frac{\pi}{2} \sqrt{\sigma_{A P} / \sigma_{E}}\right)=\eta, \eta=e \cdot\left(h / r^{2}\right)$.

where $e$ denotes load eccentricity. Detailed derivation of the counterpart formula to (4) can be found in Gere and Goodno [5]. Numerical computations produce very similar values of $\sigma_{A P}(\lambda)$ originating from equations (3) and (4) upon substitution of the same value of $\eta$. Formula (3) is preferred in design practice as being quadratic in terms of $\sigma_{A P}$ it readily delivers a closed form solution:

$$
\begin{aligned}
\sigma_{A P}(\lambda)= & \sigma_{0.2} \cdot\left[\left(\bar{\lambda}^{2}+1+\eta\right)\right. \\
& \left.-\sqrt{\left(\bar{\lambda}^{2}+1+\eta\right)^{2}-4 \bar{\lambda}^{2}}\right] /\left(2 \bar{\lambda}^{2}\right), \bar{\lambda} \\
= & \lambda / \lambda_{0.2}=\sqrt{\sigma_{0.2} / \sigma_{E}}, \lambda_{0.2}=\pi \sqrt{E / \sigma_{0.2}} .
\end{aligned}
$$

The Perry factor $\eta$ used in contemporary design practice is not used in its strictly theoretical sense but rather in quite a formal way as a kind of adjustable parametric function to describe any kind of column-load system deficiency, special loading conditions or processing, e.g. existence of a heat affected zone. Only the general functional characters of the Ayrton-Perry plot remain in connection with rigorous theoretical considerations. Various functional forms of $\eta$ 
were proposed, e.g. Robertson proposed that for columns with circular cross-sections made up of mild steel the Perry factor should be taken as a linear function of slenderness in the form $\eta=0.003 \cdot \lambda$. Dwight proposed that for aluminium columns the Perry factor should be taken in the following linear form:

$\eta=\left(c / \lambda_{2}\right)\left(\lambda-\lambda_{1}\right), \lambda_{2}=\lambda_{0.2}$.

When slenderness increases, the relative importance of column-load system deficiencies is diminished and the failure stress $\sigma_{A P}$ tends towards the Euler load $\sigma_{E}$. At the other extreme, there is accepted cut off for slenderness for stocky columns when it is accepted that failure takes place through the bulk plastic flow mode. This cut off value for particular materials and operating conditions is defined in (6) through the ratio $\lambda_{1} / \lambda_{2}$. The constant $c$ is a dominant factor for determining the level of failure stresses for plastic and elastic-plastic buckling failure modes. Its value is preset depending on experimental test results obtained for specific column operating conditions, e.g. operation in joints. British Standard BS8118 recommended the following values of $c \in<0.1,0.8>$ and $\lambda_{1} / \lambda_{2} \in<0.2,0.6>$ - see Dwight [3].

Contemporary semi-curve fitting design practices for determining buckling failure stress are adopted due to the existence of serious difficulties in adequate separation, and taking into account not only subtle and minute details of specimen imperfections, loading control process and boundary conditions but also mutual interaction of various factors. The present work is targeted at the investigation of the imperfection-interaction phenomena of the prismatic column buckling process. These phenomena express themselves the most distinctly in columns of intermediate slenderness. The direct motivation for the work was the considerably scattered experimental values of buckling loads reported in the literature, which were obtained for ostensibly identical columns.

\section{Experimental Setup and Procedures}

\section{Material}

Industrially manufactured, prismatic aluminium flats of rectangular cross-section were used for testing purposes. The one metre long flats, originating from the same production lot, were purchased from a commercial supplier. The detailed processing history of the flats was unknown and undocumented, but the flats manufacturing precision was high. Their thickness, $b=5.88 \pm 0.01 \mathrm{~mm}$, and width, $d=19.98 \pm$ $0.01 \mathrm{~mm}$, did not deviate more than $0.02 \mathrm{~mm}$ along one metre length. Three measurements of the cross-section were taken along the one metre long flat. The straightness of the flats was evaluated to be $1 / 1000 \mathrm{~mm}$, and remained better than required by the industrial standard for this type of products, i.e. $2 / 1000 \mathrm{~mm}$. In order to determine the material properties of the aluminium, dog bone specimens were manufactured with a total length of $170 \mathrm{~mm}$, gauge length of $55 \mathrm{~mm}$ and a cross-section of $\mathrm{A}=10.05 \times 5.88 \mathrm{~mm}^{2}$. An MTS 858 machine was used to carry out the material properties tests. The accuracy of the MTS 858 frame displacement and force load control was estimated to be $\Delta \mathrm{u} \cong 4 \mu \mathrm{m}, \Delta \mathrm{F} \cong 2.5 \mathrm{~N}$. The loading program consisted of three cycles of uniaxial tension-compression loading, the first two cycles remaining in the elastic range of material behaviour and the last cycle involving around $1 \%$ of plastic strain. The longitudinal and transverse strains were registered with extensometers. Elasto-plastic properties of the material were determined from prepared nominal stress-nominal strain curves, shown in Fig. 1.

The value of the linear elastic modulus was estimated to be $\mathrm{E}=63300 \mathrm{MPa}$. The behaviour of the material started to deviate away from linear elastic at $0.01 \%$ strain and $150 \mathrm{MPa}$ stress, which was accepted as the proportionality limit $\sigma_{\mathrm{P}}$. The characteristic slenderness $\lambda_{P}=64.5$, calculated from equation (2), represents the limit of the Euler buckling model validity. The conventional plastic yield stress, $\sigma_{0.2}$, at $0.2 \%$ offset permanent strain is $189 \mathrm{MPa}$ and the corresponding characteristic slenderness $\lambda_{P}=\pi \cdot\left(E / \sigma_{P}\right)^{0.5}=57.6$. The Poisson ratio varied with the level of loading, from an initial value of 0.45 to a value of 0.38 at peak load. The instantaneous unloading elasticity modulus at $1 \%$ permanent strain was determined to be $58.9 \mathrm{GPa}$. The ultimate stress $\sigma_{\mathrm{UTS}}=217.5 \mathrm{MPa}\left(\varepsilon_{\mathrm{UTS}}=6.91 \%\right)$ and fracture strain $\varepsilon_{\text {frac }} \cong$ $11.6 \%$ of the material was determined in separate tests. Comparison of the stress-strain curves and material data obtained with the information present in existing literature, led the present authors to the conclusion that the tested material is most probably A16063-T6, which is material submitted to artificial aging and stress relieving heat treatment after the extrusion process. This allows the presumption that residual stresses are at a negligible level in the as-delivered material.

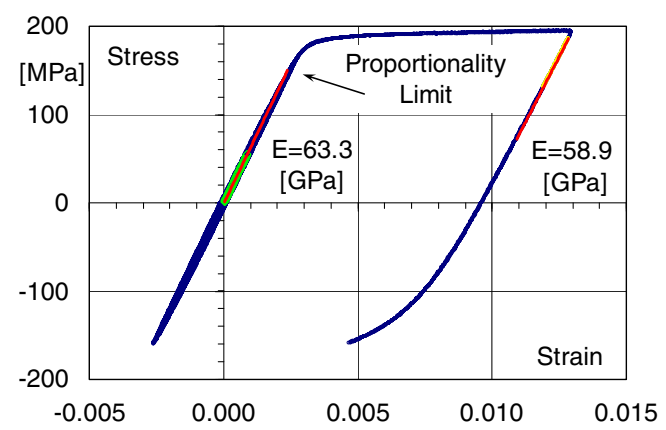

Fig. 1 Nominal stress - nominal strain curve obtained from uniaxial testing of aluminium 


\section{Buckling Test Setup}

The program of buckling tests was performed on a MTS 810 machine with a load cell capacity of $250 \mathrm{KN}$ and crossheads distance exceeding $1 \mathrm{~m}$. The photograph of the experimental set up when ready for performing buckling tests is shown in Fig. 2(a), and the scheme of measurements configuration is shown in Fig. 2(b). A fix-fix scheme of column buckling was employed as this led to a convenient mounting procedure. Prismatic flats specimens were simply clamped in machine grips at an appropriate length. During the tests only the upper grip of the testing machine was moving, attaining a displacement $u(t)$, while the lower one remained motionless. This layout called for the installation of special fixtures with a frame moving with half the vertical speed of the machine grip. Laser sensors mounted on the moving frame enabled precise measurement of the lateral deflection $2 f(t)$ of the buckling column in its mid length with an estimated accuracy of $\Delta f \cong 3 \mu \mathrm{m}$. A Teststar control unit of the MTS 810 machine allowed for the measurement and registration of displacement $u(t)$ and load $P(t)$. Both of these signals could be used as control parameters. After a skilled tune up, the Teststar control unit delivered excellent accuracy of results of displacement and force signals, estimated by the present authors to be $\Delta u \cong 3 \mu \mathrm{m}, \Delta P \cong 25 N$. In the majority of tests the displacement $u(t)$, force $P(t)$ and lateral deflection $f(t)$ signals were measured and registered. However, in order to inspect the mechanism of plastic buckling a number of tests were performed with strain gauges glued at the node and antinode of the buckling column. In total four strain gauges were glued, one on each side of the sample in two locations, see Fig. 2(b). At the node locations the bending and axial modes of deformation operated to full extent (no shearing effects appeared since $\varphi\left(x=\frac{L}{2}\right)=\frac{d y}{d x}\left(x=\frac{L}{2}\right)=0$, where $\phi$ is the angle of the neutral axis $v s$. vertical direction).
At the antinode locations only an axial mode of deformation is significant, while bending and shearing modes are negligible due to zero curvature $\kappa$ and small values of $\phi$, respectively. The buckling testing program comprised a broad range of column slenderness in order to cover all three mentioned above modes of column failure. Column specimens with the following nominal working lengths were tested: $\mathrm{L}=5$ ( $\left.\lambda_{\mathrm{ef}}=14.7\right), 10$ (29.5), 17 (50.1), 24 (70.7), 34 (100.1), 48 (141.4) and 80 (235.6) cm. Additionally, specimens with an effective slenderness of 470 were tested with a working length of $80 \mathrm{~cm}$ and a cross-sectional thickness of $3 \mathrm{~mm}$. They originated from a different stock of aluminium flats. The length between the first rows of knurling teeth of the lower and upper grips was accepted as the working length of the column specimen. It was not possible to grip the specimen with a length accuracy better than $\Delta \mathrm{L}= \pm 0.5 \mathrm{~mm}$. This variation in specimen length leads to the following variation (accuracy) in the predicted Euler loads (see formula (1)): $\lambda_{\mathrm{ef}}=70.7 \rightarrow \Delta \mathrm{P}_{\mathrm{E}}=123 \mathrm{~N} \quad\left(\mathrm{P}_{\mathrm{E}}=\right.$ $14691 \mathrm{~N}), \quad \lambda_{\mathrm{ef}}=100.1 \rightarrow \Delta \mathrm{P}_{\mathrm{E}}=43 \mathrm{~N} \quad\left(\mathrm{P}_{\mathrm{E}}=7320 \mathrm{~N}\right), \lambda_{\mathrm{ef}}=$ $141.4 \rightarrow \Delta \mathrm{P}_{\mathrm{E}}=15 \mathrm{~N} \quad\left(\mathrm{P}_{\mathrm{E}}=3673 \mathrm{~N}\right), \lambda_{\mathrm{ef}}=235.6 \rightarrow \Delta \mathrm{P}_{\mathrm{E}}=3 \mathrm{~N}$ $\left(\mathrm{P}_{\mathrm{E}}=1322 \mathrm{~N}\right)$. These values are to be compared with the internal accuracy of the machine's force measurement of 25 $\mathrm{N}$. It was also difficult to grip the specimen with a precisely planned nominal length. Due to that reason the actual lengths presented in the results section differ by up to $3 \mathrm{~mm}$ from the nominal one. The corrections were made after performing the buckling tests on the basis of the markings made on the specimens after being gripped.

\section{Testing Program}

Three series of experiments were performed. The first series consisted of a single loading unloading cycle involving the "considerable" lateral deflection of the buckling specimen.
Fig. 2 (a) Experimental setup for column stability tests on MTS 810 machine (b) Schematic view of experimental buckling configuration with measurement points

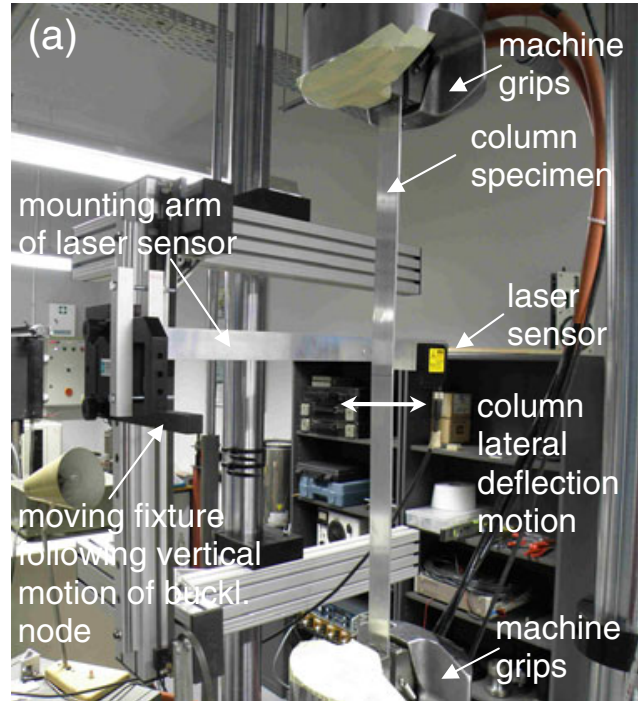

(b)

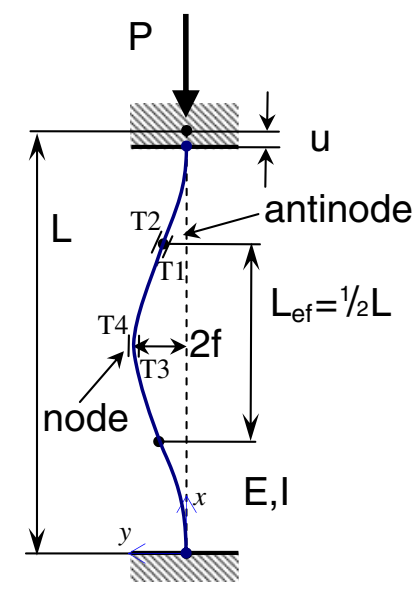


The specimen loading was displacement controlled, as force controlled experiments lead to a "catastrophic" buckling event. The results of the first series of tests served for the determination of the range of loadings for the subsequent second series of tests involving several loading cycles. The aim of this second series of tests was to investigate the behaviour of the specimens in the area of the buckling event. Various control program schemes were tried. Finally, a single test loading program comprised of force controlled loading cycles on the loading and unloading phases before the first buckling event occurred, and subsequently mixed controlled loading cycles, i.e. displacement controlled on loading and force controlled on unloading until a zero value of force was reached. The third series of tests was mixed controlled like in the second series. The difference was that additional signals were recorded during these tests, originating from strain gauges glued at the node and antinode locations. In addition several force controlled, "catastrophic", buckling tests were performed. Selected, representative results from the testing program are presented in the next section.

\section{Results}

The results of the experimental program are divided into three groups corresponding to the currently accepted three regimes of column failure, i.e. material plastic yielding, inelastic (elastic-inelastic) buckling and elastic buckling. The slenderness ratios $\lambda_{1}$ and $\lambda_{2}$ that delimit these ranges are experimentally determined for each construction material used in current design practice. In the present authors' opinion, further research is required for their precise theoretical rationalisation. For example, in the case of 6063 T6 alloy, the value of slenderness $\lambda_{2}=78$ is accepted in the design process to delimit inelastic and elastic buckling, while on theoretical grounds the validity of the linear Euler theory is delimited by the value $\lambda_{P}=64.5$. Clearly some nonexpressed explicitly additional factors, besides the elastic buckling mechanism, play a role when estimating the safety of column against failure. The analysis of graphs describing the buckling process can help in identifying these factors.

\section{Slender Column Behaviour}

The buckling and post buckling behaviour of slender columns is shown in Fig. 3. Plots of force versus lateral deflection, traditionally used in buckling analyses, are shown on the right-hand side of Fig. 3. These kinds of graphs are very good for the detection of the buckling event itself, but they do not provide clear information on the energy aspects of the buckling phenomenon as lateral deflection is just perpendicular to the operating loading force. Due to that reason, plots of axial force versus axial displacement showing the relevant energy information were prepared, as shown on the left hand side of Fig. 3. In graphs $3 \mathrm{a}-\mathrm{b}$, the buckling and post-buckling behaviour of columns of slenderness $\lambda=235.6$ is shown. The force curve (marked with dark blue circles) shows that the column buckles at the value of load (force) as predicted by the Euler elastic buckling model. The Euler load being shown in the graphs by a pink horizontal broken line. The buckling event in graph $3 \mathrm{a}$ is marked with pink vertical dot-dash line. The event can be simply and conveniently determined as an asymptote towards the $\mathrm{x}$-axis of the lateral deflection versus the axial load curve (marked with green triangles). The buckling test consisted of several loading-unloading cycles. When the lateral deflection did not reach too great a value, the loading and unloading paths were practically the same, in other words no hysteresis loop was exhibited by the column-load system. The extent of lateral deflection to which no hysteresis loop was observed, is marked in a red colour on the respective curves in graphs $3 a-b$. It can be surmised that in such situations the column buckles in the elastic range of material behaviour, and plastic deformation effects can be neglected. It should be noted that in the postbuckling phase, the buckling load remains constant as predicted by the Euler model. In graph $3 b$, a rounding off of the force curve before actually reaching the Euler load can be clearly noticed. This is a characteristic feature for column-load system imperfections.

The buckling and post-buckling behaviour of a column of slenderness $\lambda=141.4$ is shown in graphs $3 \mathrm{c}-\mathrm{d}$. This column also buckled at the value of force predicted by the Euler model with a wide, post-buckling plateau of constant force. The no hysteresis loop range of lateral deflection, marked by a red colour respective curves in graphs $3 \mathrm{c}-\mathrm{d}$, is shorter in comparison to the behaviour of the column of slenderness $\lambda=235.6$. A gentle decrease in the postbuckling force indicates that plastic deformation of the column starts to play a role. In graphs 3e-f, the buckling and post-buckling behaviour of columns of slenderness $\lambda=$ 101.3 is shown. For this slenderness conformance to the Euler model is still exhibited, as the column buckles at the force predicted by the Euler model. However, only a very short post-buckling plateau of constant force is exhibited in the region of the buckling event. For columns with slenderness $\lambda=101.3$, it was practically impossible to distinguish the range of post-buckling behaviour, in which no hysteresis loop could be observed. This means that shortly after the elastic buckling process, the plastic deformations start to play significant role, which also shows through a considerable decrease in post-buckling force. We have already highlighted the issue of control of the buckling process. In graphs $3 e-f$, the results of the controlled displacement load test, drawn with a dark blue 
Fig. 3 Buckling and post buckling behaviour of slender columns submitted to displacement controlled axial compressive load. (a-b) Column with slenderness $\lambda=235.6\left(\mathrm{~L}_{\mathrm{ef}}=40.0 \mathrm{~cm}\right)$, (c-d) Column with slenderness $\lambda=141.4\left(\mathrm{~L}_{\mathrm{ef}}=24 \mathrm{~cm}\right),(\mathbf{e}-\mathbf{f})$ Column with slenderness $\lambda=$ $101.3\left(\mathrm{~L}_{\mathrm{ef}}=17.2 \mathrm{~cm}\right)$. Results of force load controlled buckling test marked with light blue rectangles, keyed "Force ff" are overlaid on displacement load controlled test data drawn in dark blue line, keyed "Force df" (a)

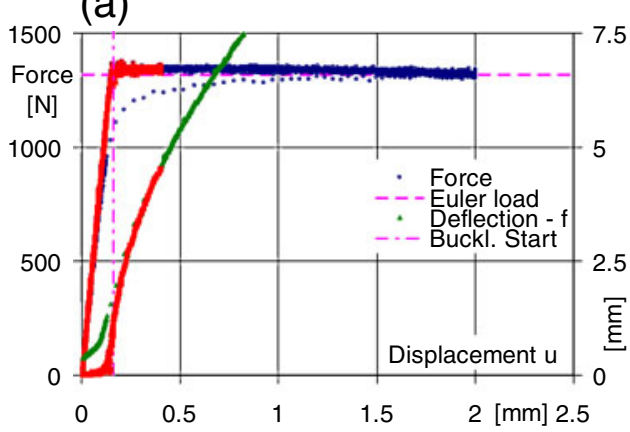

(c)

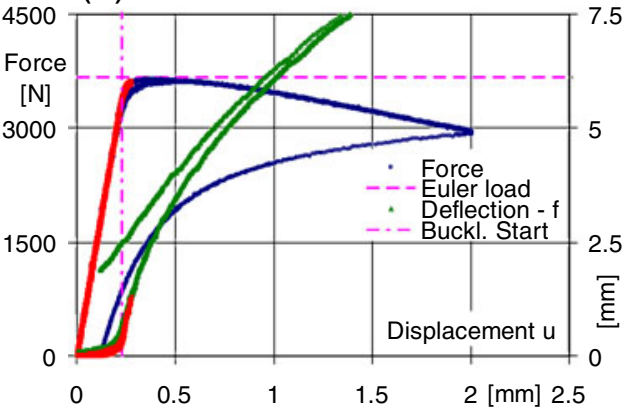

(e)

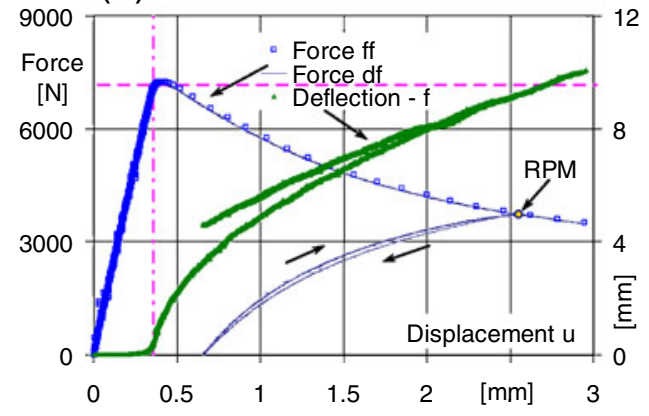

(b)

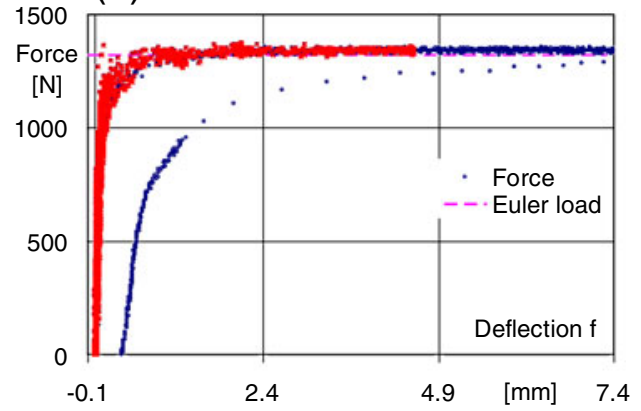

(d)

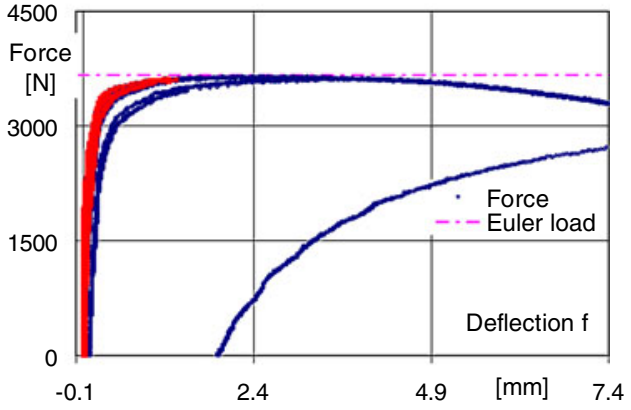

(f)

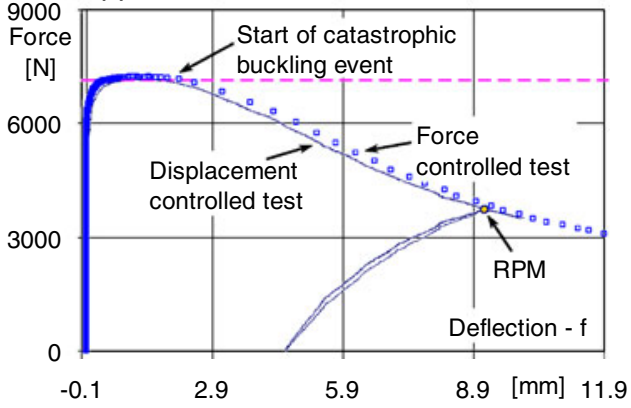

line and designated "Force df", were overlaid with force controlled buckling test data, marked with light blue rectangles and designated "Force ff". The curves are practically identical with the same pre-buckling behaviour, buckling load value and post-buckling phase. The difference is that the post-buckling phenomena in force controlled experiments cannot be easily observed, if at all. The experimental data registered in the post-buckling phase of force controlled tests were actually registered after a safety trip out of the testing machine, signifying a catastrophic event in engineering life. Graphs 3e-f provide grounds to establish laboratory tests aimed at investigating the buckling phenomenon for the purposes of engineering design, which can, and should, be performed in displacement control mode. They then are capable of delivering more information in a credible and meaningful way. This is further demonstrated in the subsequent tests. In graphs $3 \mathrm{e}-\mathrm{f}$ it can be noticed that when the buckled column is unloaded to zero force from a certain arbitrary post-buckled state and then reloaded again, the loading path closely follows that of the unloading one, showing very small hysteresis. Upon exactly reaching the force level from which the unloading process was originally initiated, the post-buckling states envelope is further followed, as if the unloading process never took place. Thus buckling columns exhibit a so called return point memory (RPM) effect. This kind of behaviour was observed for buckling columns with any slenderness index, and from whichever post-buckling state the unloading process was initiated, see also Fig. 4(a).

This observation is of great practical value. The envelope of post critical states, which can be determined simply and easily in one displacement controlled buckling test, ran to advanced values of lateral deflection, and in fact determines the post-buckling load bearing capacity of the column structure. Let us suppose that accidental (as a result of major force) or purposeful (due to some kind of assault) buckling of a column structural element occurred that did not lead to immediate structure collapse. Then the remaining capacity of 
Fig. 4 Buckling and post buckling behaviour of intermediate slenderness columns. (a-b) Force $v s$. axial displacement and force vs. lateral deflection plots for column with slenderness $\lambda=$ $72.5\left(\mathrm{~L}_{\mathrm{ef}}=12.3 \mathrm{~cm}\right)$ - elasticplastic buckling, (c) Part of loading program and loadcolumn system response for column with slenderness $\lambda=$ 72.5 before and after first buckling event $\left(t_{\text {Buckl }}=1185.5 \mathrm{~s}\right),(\mathbf{d})$ Force vs. axial displacement for column with slenderness $\lambda=$ $50.1\left(\mathrm{~L}_{\mathrm{ef}}=8.5 \mathrm{~cm}\right)$ - plastic buckling (a)

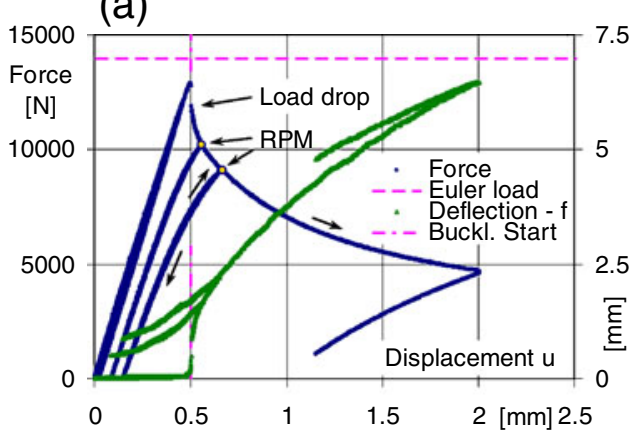

(c)

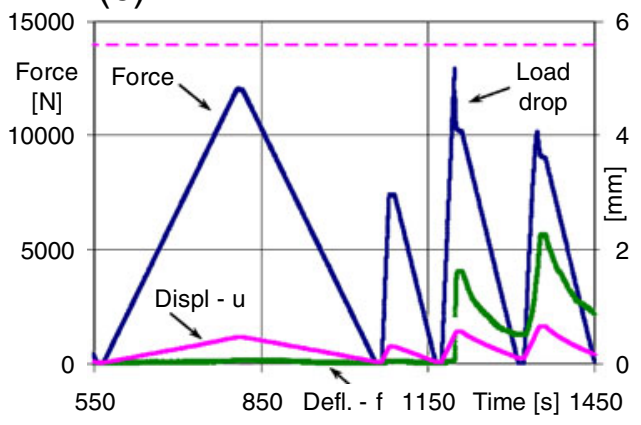

(b)

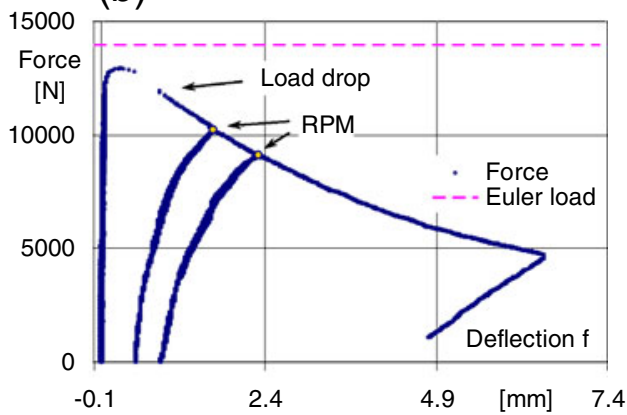

(d)

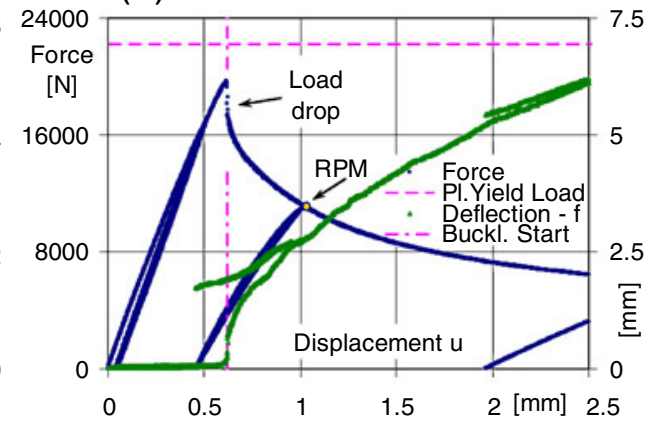

the structure to support the load after the harmful event can be simply and credibly evaluated, even if only the size of the lateral deflection amplitude in the buckling node of column structural element can be estimated. In turn, this information allows for the sound evaluation of the remaining structural safety and ability to take a decision as to whether to permit further operations within the harmed construction. The experimental results of the slender column behaviour discussed, agree well with the Euler buckling model predictions in reaching the Euler buckling load value and maintaining this value in post-buckling states. Finally, examination of graphs $3 \mathrm{~b}, 3 \mathrm{~d}$ and $3 \mathrm{e}$ show that the curving of the lateral deflection curve in the area of the buckling event is small. This allows the assessment that the aluminium flats tested were manufactured with high precision.

\section{Intermediate Slenderness Column Behaviour}

Buckling and post buckling behaviour of columns of intermediate slenderness is shown in Fig. 4. No precise definition of the expression "intermediate" slenderness can be found in literature. The common acceptance is that this is a range of slenderness situated on both sides of the slenderness value $\lambda_{0.2}$, here $\lambda_{0.2}=57.6$. Buckling design procedures allow the assertion that the range is defined by values of slenderness $\lambda \in\left\langle\lambda_{1}, \lambda_{2}\right\rangle$, also refer to the text below formula (6). When accepting this last statement, $\lambda_{1}$ should be taken as greater than 20. Force versus axial displacement and force versus lateral deflection curves obtained for columns of slenderness $\lambda=72.5$ are shown in graphs 4a-b. The markings and colours in Fig. 4 have the same meaning as those of Fig. 3, so are not explained again here. Two distinctive features can be noticed in graphs $4 a-b$, which are at odds with the Euler model predictions. First, the column buckles at smaller load than predicted by the Euler model, i.e. at $92 \%$ of the Euler load. A similar observation was already reported by numerous authors reviewed in the literature and attributed to load-column system imperfections. The presence of such imperfections in the investigated case express themselves in a characteristic way through the rounding off of the force-deflection curve just before the buckling event is noticeable, see graph $4 \mathrm{~b}$ ). Second, a drop in the buckling load occurs, practically at the very moment of the buckling event. This phenomenon is not easily noticeable in the traditional studies of buckling in the force-lateral deflection graph of $4 \mathrm{~b}$. The sign that something happens takes the form of noticeable gaps between individual experimental data points appearing in graph $4 \mathrm{~b}$, immediately after the buckling event peak load. The effect is better percieved in graph $4 \mathrm{a}$, where noticeable gaps between individual experimental data points appear immediately after the buckling event. The most obviously evidence of the buckling load drop phenomenon can be perceived in the force versus time plot of the column-load system shown in Fig. 4(c). Peak buckling load drops by $20.8 \%$ in the case of the column of slenderness $\lambda=72.5$. When the column was unloaded to zero force and then submitted to consecutive loading, it buckled at $73 \%$ of the Euler load, exhibiting the return point memory (RPM) effect discussed in the previous section. The force versus axial displacement curve obtained 
for columns of slenderness $\lambda=50.1\left(<\lambda_{0.2}=57.5\right)$ is shown in graph $4 \mathrm{~d}$. The column buckled at a smaller load then that corresponding to material plastic yield stress, i.e. at $88 \%$ of plastic yield load. The buckling load drop effect also appeared for this column, and peak buckling load dropped by $21.7 \%$. When the column was unloaded and reloaded again, it buckled at $69 \%$ of plastic yield load, exhibiting a return point memory (RPM) effect.

The buckling load drop phenomenon occurring for intermediate slenderness columns is entirely new information to the best of the knowledge of the present authors. The observation is in disagreement to conjectures/predictions made in all major buckling models of Euler, Engesser and Shanley, which predict constant or increasing values of post-buckling force in the vicinity of the buckling event. Detailed discussion of Euler, Engesser and Shanley column buckling models and their predictions can be found in Gere and Goodno [5]. The second discrepancy is that the buckling load does not reach the Euler stress or plastic yield stress at the moment of the buckling event.

\section{Anatomy of the buckling load drop phenomenon}

The reasons and mechanisms of the peculiarities of intermediate column global buckling behaviour as previously described were not clear to the present authors. Therefore a series of tests were carried out on columns with strain gauges glued at the node and antinode locations [see Fig. 2(b)] in order to obtain local information on the buckling column-load system. The registered signals from respective strain gauges are designated: $\varepsilon_{T 1}, \varepsilon_{T 2}, \varepsilon_{T 3}, \varepsilon_{T 4}$. The global and local behaviour of the intermediate slenderness column $(\lambda=72.5)$ in the region of the buckling event, where it exhibiting a load drop effect, is shown in graphs $5 \mathrm{a}$ and $5 \mathrm{~b}$, respectively. Graph $5 \mathrm{a}$ is a suitably selected detail of graph $4 \mathrm{c}$, prepared to enable its convenient comparison with the local responses of the column-load system shown in graph $5 \mathrm{~b}$. Analogous global and local responses versus time of slender columns $(\lambda=$ 141.4) in the locality of the buckling event, not exhibiting a load drop effect, is shown in graphs $5 \mathrm{c}$ and $5 \mathrm{~d}$. Strains in graphs $5 \mathrm{~b}$ and $5 \mathrm{~d}$ are depicted as positive when compressive, inconsistent with common convention but convenient for the investigation of column buckling phenomena.

The buckling event itself is recognised here with the aid of an asymptote towards the $\mathrm{x}$-axis of the lateral deflection versus axial displacement curve. The event is marked by vertical dotdash lines in all graphs of Fig. 5. In the case of intermediate slenderness columns, it is clearly seen in graph $5 \mathrm{a}$ that the buckling event corresponds to the buckling load drop event itself. Hence, in the case of intermediate columns, buckling load drop can be accepted as the initiation of the buckling process. Immediately after the initiation of the buckling event complex stress state develops, which can be reconstructed to some extent using information from the plots in Fig. 5. The basic assumption of the Euler and Ayrton-Perry models is that the stress state in the cross-section of any buckling column can be decomposed into axial compression and pure
Fig. 5 (a-b) Global and local response of intermediate slenderness column-load system $(\lambda=$ 72.5) exhibiting buckling load drop phenomenon. (c-d) Global and local response of slender column-load system $(\lambda=141.4)$ exhibiting buckling load upholding (a)

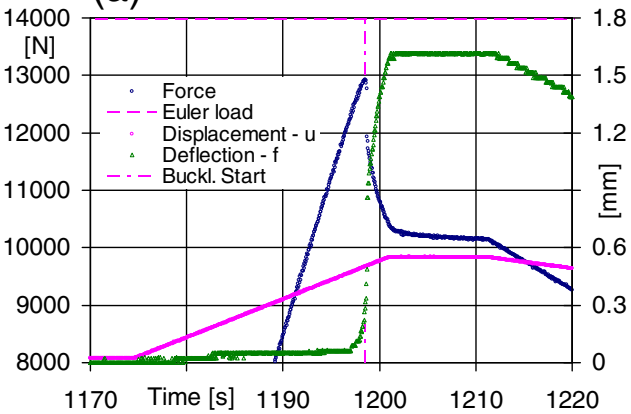

(c)

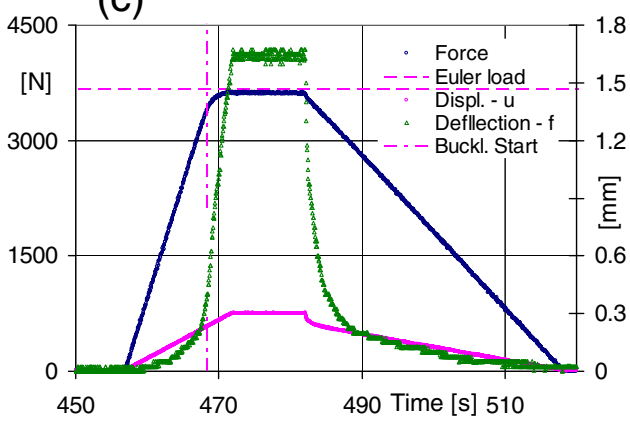

(b)

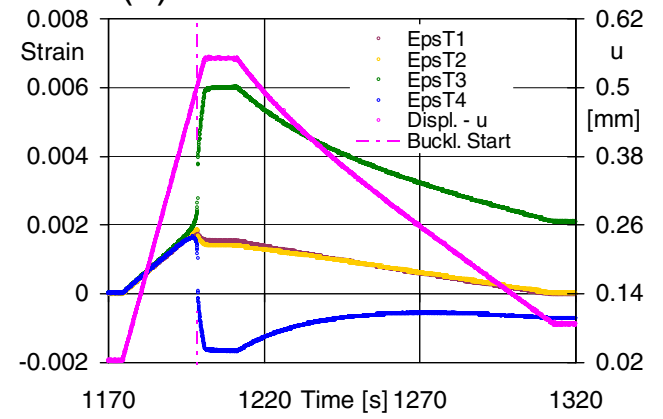

(d)

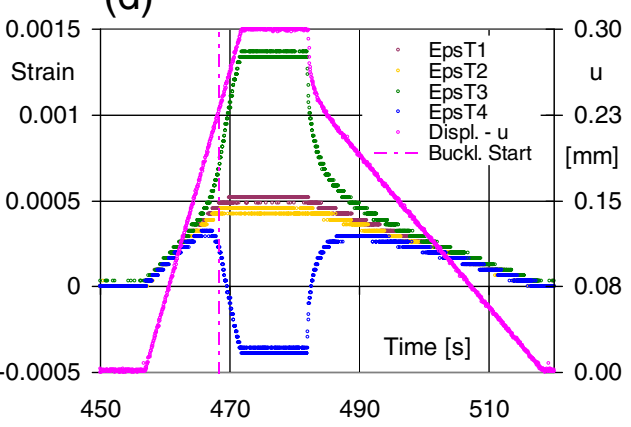


bending stress states, and that the material does not enter the inelastic range of behaviour. Then the strain in any fibre of any cross-section of the buckling column can be expressed analytically with the formula:

$$
\begin{aligned}
\varepsilon(x, z) & =\varepsilon_{a x}+\varepsilon_{b d}(x, z)=P /(E A)+\kappa(x) \cdot z, \kappa \\
& =M(x) / E I, x \in<-L, L>, z \in<-h, h>.
\end{aligned}
$$

The following analytical expressions are thus obtained for the measurement of experimental strains:

$$
\begin{aligned}
\varepsilon_{T 3} & =\varepsilon\left(\frac{1}{2} L,-h\right)=\varepsilon_{a x}-\kappa_{\max } \cdot h, \varepsilon_{T 4}=\varepsilon(L,-h) \\
& =\varepsilon_{a x}+\kappa_{\max } \cdot h ; \kappa_{\max }=-P f / E I, \varepsilon_{T 1} \\
& =\varepsilon(0,-h)=\varepsilon_{T 2}=\varepsilon(L, h)=\varepsilon_{a x} ; \kappa=0 .
\end{aligned}
$$

Eliminating $\varepsilon_{a x}$ from (7) $)_{1,2}$ with the aid of (7) 3 , one comes to the conclusion that the following relation must be fulfilled for the above assumption on stress decomposition to be valid:

$$
I=\left(\varepsilon_{T 3}-\varepsilon_{a x}\right) /\left(\varepsilon_{T 4}-\varepsilon_{a x}\right)=-1, \varepsilon_{a x}=\left(\varepsilon_{T 1}+\varepsilon_{T 2}\right) / 2 .
$$

Let us first analyse the behaviour of the slender column $(\lambda=141.4)$ shown in graphs $5 \mathrm{c}-\mathrm{d}$. The buckling event started at time $t=468.3 \mathrm{~s}$. The stable combined post-buckling stress state was formed soon thereafter whilst maintaining a constant displacement $u$-the load control parameter. The following values of strains were registered; $\varepsilon_{T 3}=0.000135$, $\varepsilon_{T 4}=0.000375, \varepsilon_{T 1}=0.00044, \varepsilon_{T 2}=0.00049$ at time $t=$ 474.5 s, i.e. in close vicinity to the buckling event. Substituting these values into equation $(8)_{1}$ results in $I_{\exp }=-1.03 \cong-1\left(\varepsilon_{a x}=0.00047\right)$. Thus the experimental data confirmed validity of assumption (7) and the underlying Ayrton Perry formula, and proved that it can be credibly used as phenomenological tool to predict the buckling behaviour of slender columns. It should be noted that a buckling load drop effect did not take place during the process of switching deformation modes, from simple axial compression to a combined stress state. Slight discrepancies in the readings of strain gauges $\mathrm{T} 1$ and $\mathrm{T} 2$ most probably originate from slight deviation from their exact antinode location. Upon unloading to zero force, all strain gauge readings returned to zero, indicating that no residual stress fields had generated as a result of the buckling process that the column experienced.

Now considering the intermediate slenderness column $(\lambda=72.5)$ behaviour as shown in graphs $5 \mathrm{a}-\mathrm{b}$. The column buckled at time $t=1198.5 \mathrm{~s}$. After the buckling load drop period, when the simple axial compression deformation mode switched to a combined stress state deformation, a stable combined post-buckling stress state was formed while the load control parameter $u$ remained constant. The following values of strains were registered: $\varepsilon_{T 3}=0.0060$, $\varepsilon_{T 4}=-0.00166, \varepsilon_{T 1}=0.00140, \varepsilon_{T 2}=0.00153$ at time $t=$ 1205.35 , i.e. in close vicinity to the buckling event after a buckling load drop took place. Substituting these values into (8) gives $I_{e x}=-1.45 \neq-1 \quad\left(\varepsilon_{a x}=0.00147\right)$. This indicates that in the case of intermediate slenderness columns, exhibiting the buckling load drop phenomenon, the Ayrton Perry formula cannot be accepted as a suitable phenomenological modelling tool to predict their behaviour, even as a rough approximation. In spite of the appearance of only minute plastic strains being at the level of 0.002 (see Fig. 5(b)), that is the value accepted as offset defining macroscopic plastic strains. A complex stress state is formed as a result of the switching process of deformation modes involving a buckling load drop phenomenon, largely asymmetric in the $z$ direction of the crosssection. An even more asymmetric, residual inelastic strain field is formed when the buckled column is subsequently unloaded to zero force. The following values of strains were registered: $\varepsilon_{T 3}=0.00208, \varepsilon_{T 4}=-0.00072, \varepsilon_{T 1}=0.0000$, $\varepsilon_{T 2}=0.0000$ at time $t=1317.7 \mathrm{~s}(P=0)$. Substituting these values into (8) $)_{1}$ results in $I_{e x}=-2.9 \neq-1\left(\varepsilon_{a x}=0.0\right)$.

Further research is required to fully grasp the essential features of the buckling load drop effect, and in order to develop an appropriate theoretical model in which the presence of a minute, non-symmetric inelastic strain field must be taken into account. This would probably involve using finite element code equipped with an advanced load stepping algorithm to overcome the problem which might appear with the passage of sudden softening of the system. in addition to further experimental work. At present, it can be stated that the interaction mechanism of load-column system imperfections with changing modes of deformation is responsible for a qualitative change of the intermediate slenderness column's buckling behaviour. It expresses itself in the form of a buckling load drop and the formation of a highly asymmetric residual stress field and also as the permanent deformations that arise during the small lateral deflection of the intermediate slenderness column's buckling process, though at the buckling node (locally) these reach a value of $0.2 \%$ which in global terms are hardly noticeable. Residual displacement upon column unloading is $u_{r e s}=0.09 \mathrm{~mm}$, and this is to be referred to a specimen with a total length of $L=246 \mathrm{~mm}$. Residual deflection is $\mathrm{f}_{\text {res }}=0.51 \mathrm{~mm}$, and when referred to $L=246 \mathrm{~mm}$ it gives a deviation from straightness remaining within the level of standard manufacturing tolerances for commercially produced aluminium flats requirement of less than 2/1000. A researcher or engineer, unaware that an ostensibly virgin column gripped in the testing machine had already undergone a cycle of slight buckling (inescapably involving a buckling load drop), would notice with surprise that the 
Fig. 6 Buckling and post buckling behaviour of stocky column with slenderness $\lambda=30.3$, $\left(\mathrm{L}_{\mathrm{ef}}=5.15 \mathrm{~cm}\right)$ (a)

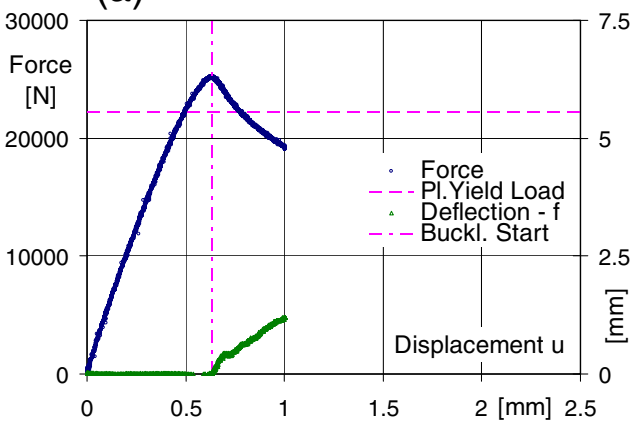

(b)

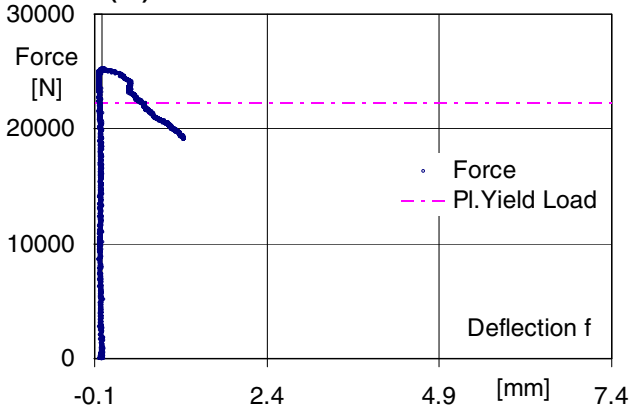

column would exhibit a buckling load at a level of $73 \%$ of the Euler load and not the expected buckling load of $92 \%$ of the Euler load. The authors would dare to suggest that the buckling load drop phenomenon discovered can help clarify the hardly explainable, considerably scattered experimental values of buckling loads reported in the literature for intermediate slenderness columns.

\section{Behaviour of Stocky Columns}

The buckling and post-buckling behaviour of stocky columns is shown in Fig. 6. Force versus axial displacement and force versus lateral deflection curves obtained for column of slenderness $\lambda=30.3$ are shown in graphs $6 \mathrm{a}-\mathrm{b}$. The column significantly enters the material bulk plastic yield deformation regime well before the column starts to buckle, which can be clearly observed in graph 6a. In fact the column started to buckle at a load very close to the ultimate stress of the material. This result conforms to that reported in the literature. Similarly as in the case of slender columns, no drop of buckling load is observed for stocky columns.

\section{Discussion and Concluding Remarks}

The collective information on the failure of aluminium columns subject to an axial compressive load gathered within the present experimental program is shown Fig. 7. The background information on existing buckling model predictions and design practices is also presented. The stresses at which columns of various slenderness failed are marked with green and yellow triangles. Those cases where slender column failure took place by elastic buckling, at loads as predicted by the linear Euler model, are indicated in Fig. 7 by a continuous red line. The circular markings in dark blue and green, labelled with numbers, indicate certain characteristic points important from a theoretical and/or design practice point of view. The marking labelled 3, located at slenderness $\lambda_{P}-\mathrm{cf}$. (2), indicates the limit of the linear Euler model's prediction validity. The horizontal dark blue line is drawn at the material plastic flow yield limit stress, commonly accepted in design practice as the failure stress for stocky columns. The marking labelled 2 , located at slenderness $\lambda_{0.2}$ - cf. (5), that is lying on the crossing point of the Euler model extension and material plastic flow yield limit stress, indicates the reference point commonly accepted as loosely defining the transition region between slender and stocky columns. The region of intermediate slenderness columns is more precisely defined by slenderness $\lambda_{1}$ and $\lambda_{2}$ and indicated with markings labelled 1 and 2 . In present design practice, this region is determined with the aid of experimental tests and curve fitting procedures. The reason for that is an insufficient and inadequate theoretical base.

The common understanding is that columns can be divided into three classes: slender which exhibit elastic buckling failure mechanisms, intermediate which display elasticplastic or plastic buckling failure mechanisms and stocky presenting bulk material plastic flow before possible buckling. The theoretical basis for the description of the behaviour of columns at the point of failure from the first class is relatively well developed. It is through the Ayrton Perry model, which contains parameters enabling imperfections of the columnload system to be taken into account. The theoretical basis for

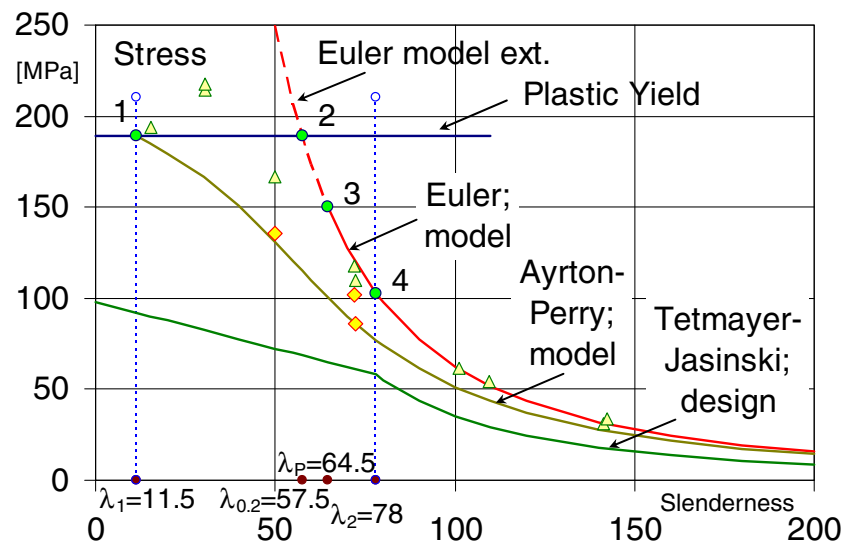

Fig. 7 Current data on failure loads of columns subjected to compressive axial loads-overall buckling stresses. The solid lines indicate modelling predictions and design curves 
the description of the behaviour of the columns at failure belonging to the third class is also well developed, being the classic theory of plasticity. The behaviour of intermediate slenderness columns at failure at present constitutes an open scientific problem awaiting proper theoretical elaboration. It has been shown that the Ayrton Perry model cannot be accepted, even as rough approximation for describing the behaviour of columns with intermediate slenderness. In the task of elaborating the buckling model for intermediate slenderness columns, mutual interaction-imperfection coupling effects must be taken into account. These effects play an essential role, as reported here for the first time, in the phenomenon of the column buckling load drop. As a result proper theoretical predictions of the size of such a buckling load drop would be possible. The scale of this effect can be observed in Fig. 7, where the vertical distance between the respective green-yellow triangle markings and red-yellow diamond markings indicates a drop of buckling load after the occurrence of minute primary buckling in the column. The diamond markings also indicate the stress at which buckling is going to take place in the minutely buckled column upon its subsequent reloading. The design practice approach recommended by British Standards, based on the AyrtonPerry model, is shown by an olive line in Fig. 7. The predicted buckling loads for intermediate slenderness columns are obtained upon the substitution into the AyrtonPerry formula (5) of a sufficiently large value of a Perry factor parameter, defined here as $\eta=0.0055$. The slenderness $\lambda_{1}=11.5$ arbitrarily delimits the "bulk plastic flow" and "plastic buckling" region - see also Dwight [3] section 5.4.2 $\left(\lambda_{0}=57.5, C=0.2\right)$. When safety factors are included in the Ayrton Perry modelling prediction, the values of allowable design stresses are obtained. The dark green curve shows the admissible stresses recommended by the Aluminium Association for the alloy Al60063-T6, i.e. Tetmayer-Jasinski modelling predictions divided by factors of safety recommended as $n=1.65$ for plastic flow region ("nonexistent" here) and $n=1.77$ for plastic buckling $\left(\lambda \in<\lambda_{1}=0, \lambda_{2}=78>\right)$ and the elastic buckling region $\left(\lambda>\lambda_{2}=78\right)$.

The results of the present study indicate that running column buckling tests under displacement control is worthy of being adopted as common practice. Since then, the envelope of column post-buckling states can be conve- niently determined. This information in turn allows for the quick and reliable estimation of the safety of such a column structure, upon taking advantage of the RPM property, which has undergone some accidental or deliberate damage. For example this could be as a result of foreign object damage in the form of column limited buckling when under operational load.

Acknowledgements The authors acknowledge the financial support provided through the Polish Ministry of Science and Higher Education Grants: No PB N50104131/2765, "The analysis of critical and postcritical states of structures under conservative and non-conservative loads. Experimental and theoretical investigation, verification of existing design codes"-SI and AZ, and Nr N N501 078435 "Elaboration of monitoring methodology of damage development and its localization in low alloyed steels and aluminium alloys"-AZ.

Open Access This article is distributed under the terms of the Creative Commons Attribution Noncommercial License which permits any noncommercial use, distribution, and reproduction in any medium, provided the original author(s) and source are credited.

\section{References}

1. Barbero E, Dede E, Jones S (2000) Experimental verification of buckling-mode interaction in intermediate-length composite columns. Int J Solids Structures 37:3919-3934

2. Domokos G, Holmes P, Royce B (1997) Constrained euler buckling. J Nonlinear Sci 7:281-314

3. Dwight J (1999) Aluminium design and construction. 2nd edn. Taylor \& Francis Group

4. Garikipati K, Göktepe S, Miehe C (2008) Elastica-based strain energy function for soft biological tissue. J Mech Phys Solids $56: 1693-1713$

5. Gere J, Goodno B (2009) Mechanics of materials, 7th edn. Cengage Learning, Stamford

6. Kissell J, Ferry R (2002) Aluminium structures, a guide to their specifications and design, 2nd edn. Wiley, New York

7. Kuznetsov V, Levyakov S (2002) Complete solution of the stability problem for elastica of Euler's column. Int $\mathrm{J}$ NonLinear Mechanics 37:1003-1009

8. Magnusson A, Ristinmaa M, Ljung C (2001) Behaviour of the extensible elastica solution. Int J Solids Structures 38:8441-8457

9. Mazzilli C (2009) Buckling and post-buckling of extensible rods revisited: a multiple-scale solution. Int J Non-linear Mechanics 44:200-208

10. Simitses G, Hodge D (2006) Fundamentals of structural stability. Elsevier 